\title{
Toxic Chemicals and their Neutralising Agents in Porous Media
}

\author{
Mohit Dalwadi ${ }^{1} \dagger$, Elizaveta Dubrovina ${ }^{2}$, Almut Eisenträger ${ }^{1}$, Alpha \\ Lee $^{1}$, Joseph Maestri ${ }^{2}$, Bartlomiej MatejczyK ${ }^{3}$, Doireann O'Kiely ${ }^{1}$, Megan \\ StAmper $^{4}$, and Stuart ThOMSON ${ }^{1}$ \\ ${ }^{1}$ University of Oxford \\ ${ }^{2}$ Imperial College London \\ ${ }^{3}$ Warsaw University \\ 4 University of Cambridge
}

(Communicated to MIIR on 14 May 2021)

Study Group: ESGI 100. 7-11 April 2014, Oxford, UK

Communicated by: Chris Breward

Industrial Partner: UK Government Decontamination Service

Presenter: Anthony Arkell and Hasmitta Stewart

Team Members: Anthony Arkell, UK Government Decontamination Service; Tomass Bernots, Imperial College London; Chris Breward, University of Oxford; Maria Crespo Moya, Universidad Complutense de Madrid; Mohit Dalwadi, University of Oxford; Liam Dempsey, Imperial College London; Elizaveta Dubrovina, Imperial College London; Almut Eisenträger, University of Oxford; Chris Farmer, University of Oxford; Cameron Hall, University of Oxford; Ian Hewitt, University of Oxford; Stefanie Hirsch, University of Vienna; Tania Khaleque, University of Oxford; Andrew Lacey, Heriot-Watt University; Alpha Lee, University of Oxford; Joseph Maestri, Imperial College London; Bartlomiej Matejczyk, Warsaw University; Jamie Nutter, Imperial College London; John Ockendon, University of Oxford; Doireann O'Kiely, University of Oxford; Bogna Pawlowska, University of Exeter; Carmel Ramwell, The Food \& Environment Research Agency; Megan Stamper, University of Cambridge; Hasmitta Stewart, UK Government Decontamination Service; Stuart Thomson, University of Oxford.

Industrial Sector: Government

Tools: Fluid Dynamics, Capillary Action, PDEs, Mass Transfer

Key Words: D'Arcy's Law; Richards' Equation; Contamination

MSC2020 Codes: 76

$\dagger$ Corresponding author: dalwadi@maths.ox.ac.uk 


\section{Summary}

The UK Government Decontamination Service (UKGDS) advise Government on the national capability for the decontamination of buildings, infrastructure, transport and the open environment. Additionally, UKGDS are a source of expertise in the event of chemical, biological, radiological or nuclear (CBRN) incidents, and in the major release of hazardous materials. In the first part of the report, the study group derive and solve mathematical models to investigate the depth to which toxic chemicals may seep into dry porous substrates. This involved applying Darcy's law to an unsaturated porous medium for a sharp interface, and Richards' equation for a diffuse interface. In the second part, the group investigated the key cleanser properties to consider when choosing decontaminants for the neutralisation process between a decontaminant and an imbibed chemical, where the reaction product is soluble and insoluble in the cleanser phase. This involved solving reaction-diffusion equations with fixed and moving boundaries. 


\section{Report author}

Mohit Dalwadi (University of Oxford), Elizaveta Dubrovina (Imperial College London), Almut Eisenträger (University of Oxford), Alpha Lee (University of Oxford), Joseph Maestri (Imperial College London), Bartlomiej Matejczyk (Warsaw University), Doireann O'Kiely (University of Oxford), Megan Stamper (University of Cambridge), Stuart Thomson (University of Oxford)

\section{Executive Summary}

The UK Government Decontamination Service advises central Government on the national capability for the decontamination of buildings, infrastructure, transport and open environment, and be a source of expertise in the event of a chemical, biological, radiological and nuclear (CBRN) incident or major release of HazMat materials. The study group constructed mathematical models to describe the depth to which a toxic chemical may seep into an initially dry porous substrate, and of the neutralisation process between a decontaminant and the imbibed chemical.

The group recognised that capillary suction was the dominant process by which the contaminant spreads in the porous substrate. Therefore, in the first instance the absorption of the contaminant was modelled using Darcy's law. At the next level of complication a diffuse interface model based on Richards' equation was employed. The results of the two models were found to agree at early times, while at later times we found that the diffuse interface model predicted the more realistic scenario in which the contaminant has seeped deeper into the substrate even in the absence of further contaminant being supplied at the surface.

The decontamination process was modelled in two cases; first, where the product of the decontamination reaction was water soluble, and the second where the reaction product formed soluble in the contaminant phase and of similar density. These simple models helped explain some of the key physics involved in the process, and how the decontamination process might be optimised. We found that decontamination was most effective in the first of these two cases.

The group then sought to incorporate hydrodynamic effects into the reaction model. In the long wavelength limit, the governing equations reduced to a one-dimensional Stefan model similar to the one considered earlier. More detailed approximations and numerical simulations of this model were beyond the scope of this study group, but provide an entry point for future research in this area.

\section{Version 1.1}

September 22, 2014

iv +27 pages 


\section{Contents}

1 Problem description 1

2 Contaminant moving into porous medium 2

2.1 One-dimensional problem . . . . . . . . . . . . . . . . 2

2.2 Sharp interface model . . . . . . . . . . . . . . . . 2

2.3 Diffuse interface model . . . . . . . . . . . . . . . . . . . . . . . 4

2.4 Porous flow in OpenFOAM . . . . . . . . . . . . 6

3 Reaction and decontamination $\quad 8$

3.1 Introduction . . . . . . . . . . . . . . . 8

3.2 Decontamination: water-soluble products . . . . . . . . . . 9

3.3 Decontamination: oil-soluble products . . . . . . . . . . . 15

3.4 Conclusion . . . . . . . . . . . . . . . . . 18

4 A two-dimensional flow and reaction model for decontamination 19

4.1 Model overview and governing equations . . . . . . . . . . . . . 19

4.2 Non-dimensionalisation . . . . . . . . . . . . . . . . . . . 22

4.3 Long-wave asymptotics . . . . . . . . . . . . . . . . . . . 23

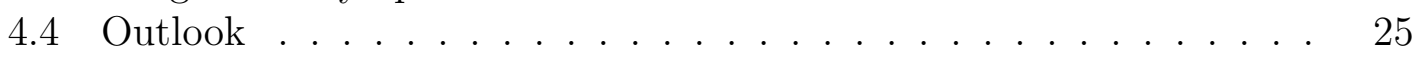

5 Conclusions and outlook $\quad 25$

$\begin{array}{ll}\text { Bibliography } & 27\end{array}$ 


\section{Problem description}

(1.1) The UK Government Decontamination Service (GDS) provides advice, guidance and assistance on decontamination related issues to responsible authorities in their contingency planning for, and response to, chemical, biological, radiological and nuclear (CBRN) and major HazMat incidents. They seek to build a better understanding of the interaction of toxic chemicals and their neutralising agents on a variety of different material surfaces. There are a number of different scenarios of interest including a few possible mechanisms of contaminant spread. We focused initially on distilling the Service's most pressing concerns into a single tractable model problem.

(1.2) The model which we felt best captured the main concerns of the GDS can be described as follows. We imagine a scenario in which a large chemical spill has occurred over a flat porous substrate. This toxic chemical will, over time, sink down into the void fraction of the porous bed. After some finite time, the decontamination service will be given the go-ahead from police and other emergency services to begin their initial clean-up and can immediately remove any toxic substance which still lies above ground. The concern at this stage is that there will remain some contaminant hidden in the pore space of the substrate. To counter this, a neutralising agent (decontaminant) will be applied at the surface in order to try to neutralise all of the remaining toxic chemical lying within the porous bed.

(1.3) We proceed with two main aims: firstly, to discover the depth to which a toxic chemical may seep into an initially dry porous bed and, secondly, to understand if it is possible to neutralise the full depth of this remaining toxic chemical by applying a neutralising agent to the surface (Fig. 1). In progressing towards these aims we have broken the problem down into two sections:

1. the initial phase: a model describing contaminant leaking into a dry porous medium;

2. the reaction phase: a description of the neutralisation of the toxic chemical via the application of a decontaminant at the surface.

We propose that this secondary, reaction phase may take one of two possible forms depending on whether the product is soluble in the oil-based contaminant phase or in the aqueous decontaminant phase. This final segmentation, based on reaction product, is motivated by a belief that the nature of the product will help determine the reaction dynamics.

(1.4) The GDS were very clear in wishing our model to remain as generalised as possible, allowing for the extrapolation of results to a wide variety of different materials. It is hoped that a better understanding of the most important physical parameters in the problem can perhaps inform the selection of a limited number of laboratory investigations. 


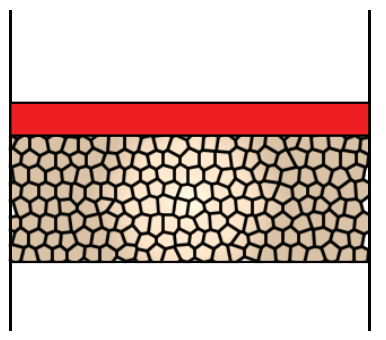

spillage

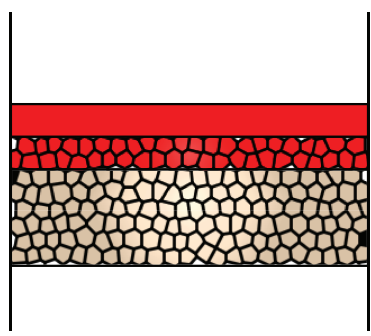

adsorption

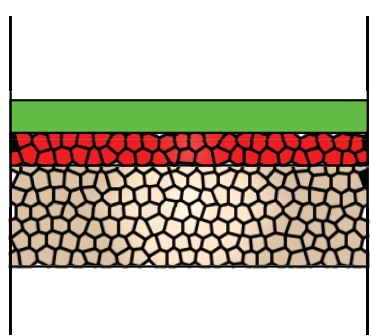

decontamination

Figure 1: Overview of the main modelling steps.

\section{Notation}

(1.5) Dimensional variables are denoted with hats $\left(^{\wedge}\right)$, to distinguish them from the dimensionless variables used throughout most of the report.

\section{Contaminant moving into porous medium}

\subsection{One-dimensional problem}

(2.1) The problem under consideration is that of a large puddle of liquid seeping into a porous material. As such, we make the simplifying assumption that the interface is approximately flat far from the puddle edge and consider a one-dimensional problem only. At time $\hat{t}=0$ a layer of fluid of dimensional height $\hat{h}_{0}$ sits at rest on the surface of a porous material. For $\hat{t}>0$ the fluid will move into the porous material under the combined action of gravity and surface tension. The dimensionless setup is illustrated in Fig. 2.

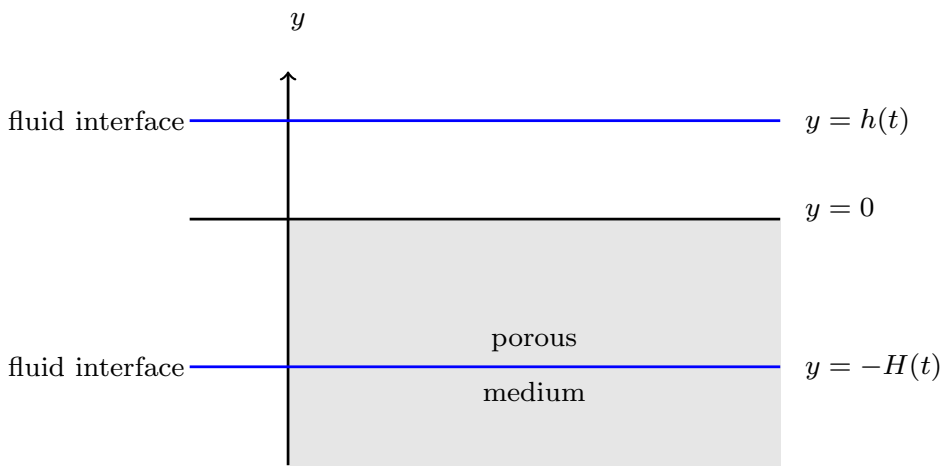

Figure 2: One-dimensional sharp interface model of contaminant moving into a flat porous medium.

\subsection{Sharp interface model}

(2.2) As a first approximation we assume that there is a sharp interface at $y=-H(t)$, above which the material is completely saturated and below 
which it is completely unsaturated. Flow through a porous medium is given by Darcy's law, which in 1D reads

$$
v=-\frac{T k \rho g}{\mu a}\left[\frac{P}{\rho g a} \frac{\partial p}{\partial y}-1\right]
$$

where $v$ and $p$ are dimensionless velocity and pressure, $g$ is acceleration due to gravity, $T$ and $P$ are typical time and pressure scales (to be determined), $k$ is the permeability of the porous medium, $\mu$ and $\rho$ are the viscosity and density of the liquid respectively, and lengths have been scaled with the typical pore radius, $a$.

(2.3) The (dimensionless) pressure inside the porous medium is governed by

$$
\frac{\partial^{2} p}{\partial y^{2}}=0
$$

with hydrostatic pressure in the liquid above the surface giving the condition

$$
p=-\frac{\rho g a}{P} h \quad \text { on } \quad y=0,
$$

and a pressure jump across the interface in the porous medium giving

$$
p=-\frac{2 \gamma}{a P} \cos (\theta) \quad \text { on } \quad y=-H(t)
$$

where $\gamma$ is surface tension at the air-liquid interface, $\theta$ is the contact angle defined as the angle between the solid surface and fluid interface measured inside the fluid and $a$ is a typical pore size in the porous material. We consider here the worst case scenario of $\theta \rightarrow 0$, corresponding to a fully wetting solid. The condition on $y=-H(t)$ motivates the scaling choice $P=2 \gamma / a$. Thus

$$
p(y)=\frac{y}{H}(1-G h)-G h,
$$

with the Bond number, $G=\rho g a / P$, giving the ratio of gravity to surface tension effects. A solution for the case of surface-tension only may be found in [1], while a solution for the case of gravity only is derived in [2]. For liquids of interest to the GDS interacting with various types of concrete, this ratio is typically in the range $10^{-10}-10^{-3}$. Inserting the pressure (2) into Darcy's law (1), performing a flux balance at $y=0$ and applying a kinematic boundary condition on $y=-H(t)$ then yields

$$
\dot{h}=-\phi \dot{H}=\frac{k \rho g T}{\mu \hat{h}_{0}}\left(\frac{1}{H}\left(h-G^{-1}\right)+1\right),
$$

where a dot $\left({ }^{\circ}\right)$ denotes a time-derivative and $\phi$ is the void fraction of the porous material. A natural scaling for time in this problem is thus $T=$ 
$a \mu / k \rho g$. The above equations may be integrated to give implicit expressions for $H$ and $h$, namely

$$
\begin{aligned}
t(H) & =-\frac{\phi}{(1-\phi)^{2}}\left[(1-\phi) H-\left(1-G^{-1}\right) \log \left(1+\frac{1-\phi}{1-G^{-1}} H\right)\right], \\
h & =h_{0}-\phi H,
\end{aligned}
$$

where $h_{0}=\hat{h}_{0} / a$. We thus have two dimensionless parameters in the problem, the Bond number $G$ and the ratio $h_{0}$ of initial fluid depth to typical pore radius.

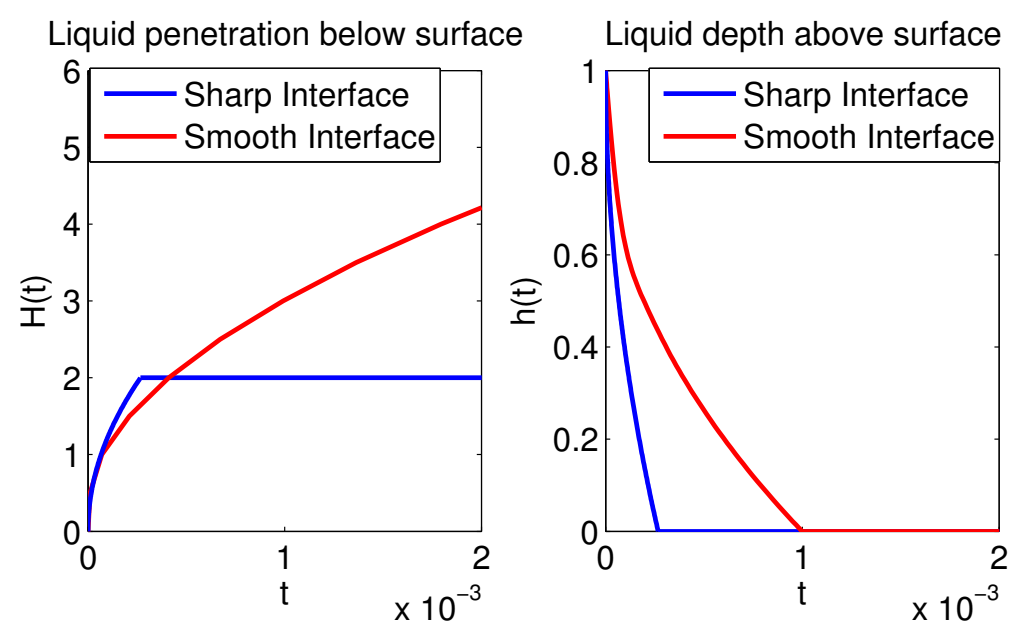

Figure 3: Plots of height of liquid above porous material surface $h(t)$ and depth reached by liquid below surface $H(t)$ for $G=2.67 \times 10^{-4}$, $\phi=0.5, h_{0}=1$. Blue lines correspond to results from the sharp interface model $(\S 2.2)$, red lines correspond to a diffuse interface model $(\S 2.3)$.

(2.4) The depths of liquid above $(h)$ and below $(H)$ the surface have been plotted in blue in Fig. 3. At early times the liquid flows into the porous medium and the height of liquid above the surface changes appropriately. Once the height of liquid above the surface reaches 0 , the depth below the surface remains fixed at $1 / \phi$.

\subsection{Diffuse interface model}

(2.5) In reality, there is not a sharp interface between fully saturated and unsaturated regions in the porous material - rather there is a saturation gradient. When surface tension dominates the system $(G \ll 1)$, the saturation is governed by Richard's Equation

$$
\phi \dot{\psi}=\frac{T \hat{D}_{0}}{\hat{h}_{0}^{2}} \frac{\partial}{\partial y}\left[D(\psi) \frac{\partial \psi}{\partial y}\right] \quad \text { in } \quad y<0,
$$


where $\psi \in[0,1]$ is saturation, $\hat{D}_{0}=k p_{c} / \mu$ and $D(\psi)$ is a dimensionless diffusion coefficient. To allow easy comparison between the sharp and smooth interface models, we use the scaling $T$ introduced in $\S 2.2$ so that the prefactor on the right-hand side becomes simply $h_{0}^{-2} G^{-1}$. We pose a general power law diffusivity of the form

$$
D(\psi)=\frac{n}{2} \psi^{n},
$$

where $n$ is material-dependent and to be determined experimentally. For simplicity we consider the case $n=1$ in this report. An alternative formulation for $D(\psi)$ and corresponding short and long time similarity solutions may be found in [1].

(2.6) At sufficiently early times (when some fluid remains in the region $y>0$ ), the porous material must remain fully saturated at the surface and the saturation profile decays to 0 far from the surface, i.e.

$$
\begin{aligned}
& \psi=1 \quad \text { on } \quad y=0 \\
& \psi \rightarrow 0 \quad \text { as } \quad y \rightarrow-\infty .
\end{aligned}
$$

Furthermore, the flux of liquid through the surface must be balanced by a change in the level of the liquid above the surface, i.e.

$$
\dot{h}=G^{-1} D(\psi) \frac{\partial \psi}{\partial y} \quad \text { on } \quad y=0 .
$$

Once the value of $h$ reaches zero there can be no more flux across $y=0$, so the boundary conditions become

$$
\begin{aligned}
& \frac{\partial \psi}{\partial y}=0 \quad \text { on } \quad \psi=1, \\
& \psi \rightarrow 0 \quad \text { as } \quad y \rightarrow-\infty \\
& \dot{h}=0 .
\end{aligned}
$$

(2.7) This system of equations was solved numerically by truncating the domain at $y=-L$ and using the method of lines; discretizing in $y$ using a secondorder finite difference scheme and integrating the resulting system of ODEs numerically using MATLAB's built-in function ode45. The saturation profile in the region $y<0$ is plotted at different times in Fig. 4. As time increases the contaminant penetrates deeper into the porous medium and the saturation profile spreads out more.

(2.8) The nonlinear diffusion imposed by $D(\psi)=\psi / 2$ causes the saturation profile to have compact support. That is, at every time $t$ there is some value of $y$ below which $\psi \equiv 0$. From this, a profile of the depth reached by the liquid as a function of time $H(t)$ may be extracted. This is plotted in red 
in Fig. 3 along with the height $h(t)$ above the material surface. Observe that at early times the sharp and diffusive interface models predict similar behaviour. However, in the diffusive interface case, the liquid continues to move deeper into the material even when there is no more liquid coming in at the surface.

(2.9) From this discrepancy we conclude that the more physically realistic model to use (when approximating the depth reached by a contaminant in a porous material), is a diffuse interface model with an appropriate choice of $D(\psi)$.

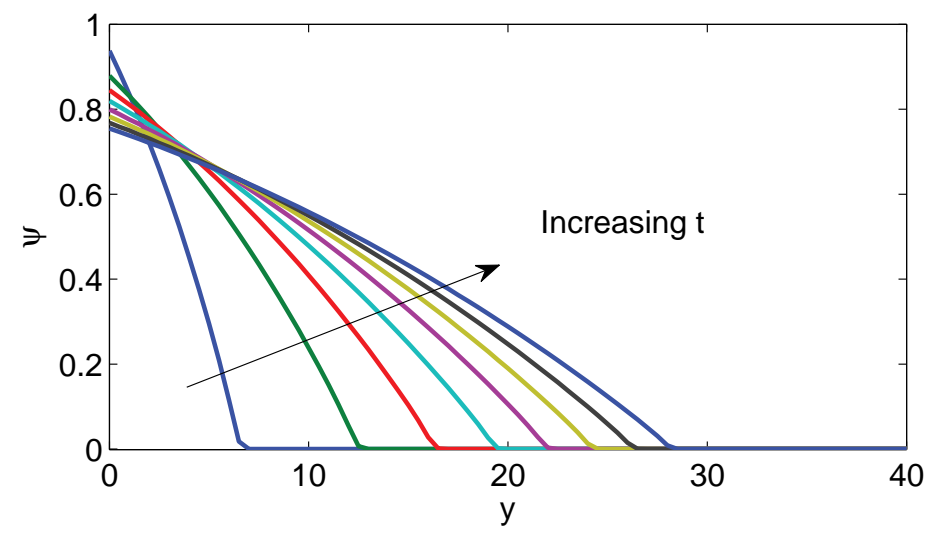

Figure 4: Saturation profiles of contaminant in porous material $\psi(y)$ for different times, for $G=2.67 \times 10^{-3}, \phi=0.95$.

\subsection{Porous flow in OpenFOAM}

(2.10) To consider extensions of the model presented above, perhaps allowing for higher dimensions or more sophisticated geometries, it is necessary to consider numerical simulation. We began to test the capabilities of the computational fluid dynamics library OpenFOAM [3]. This library would also allow future model extensions e.g. chemical reactions. The model employed for these tests differs from that in the previous sections, but describes a similar physical situation. It would be necessary in future work to attempt to work towards a clearer understanding of the differences between these two models.

(2.11) In OpenFOAM, a porous medium is modelled by attenuating the time derivative and by adding a sink term to the Navier-Stokes equations

$$
\frac{\partial}{\partial t}\left(\phi \rho u_{i}\right)+u_{j} \frac{\partial}{\partial t}\left(\rho u_{i}\right)=-\frac{\partial p}{\partial x_{i}}+\frac{\partial \pi_{i, j}}{\partial x_{j}}+S_{i}
$$

The value of $\phi$ should be between 0 and 1, where the latter is a complete porosity. The source term, $S_{i}$, is composed of two parts, a viscous loss term 
and an inertial loss term, creating a pressure drop that is proportional to the velocity and velocity squared, respectively,

$$
S_{i}=-\left(\mu D_{i, j}+\frac{1}{2} \rho\left|u_{i}\right| F_{i, j}\right) u_{i}
$$

This equation is known as the Darcy-Forchheimer equation with Forchheimer coefficient matrices $F$ and $D$. In the case of simple homogeneous porous media it becomes

$$
S_{i}=-\left(\mu D+\frac{1}{2} \rho\left|u_{i}\right| F\right) u_{i}
$$

(2.12) Using these equations we are able to run simulations of fluid penetration in porous media. We prepared a few examples using a simple 2D geometry including a porous layer and a layer of contaminant on top. We tracked the saturation level of sulfur mustards into concrete for different times (Fig. 5, 6).

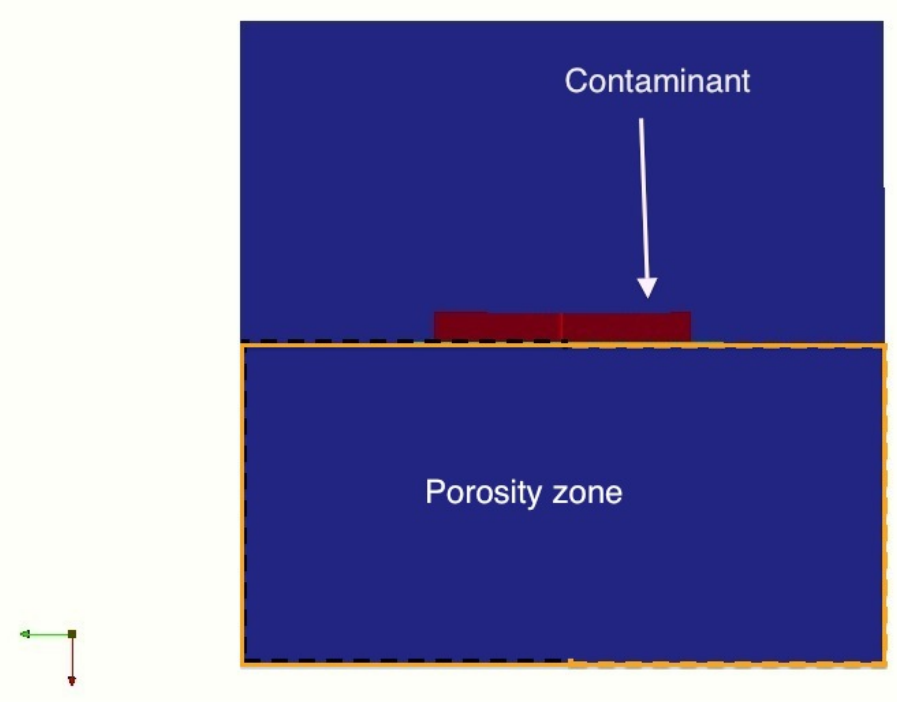

Figure 5: Initial condition $(t=0)$ for the OpenFOAM simulation of contaminant flow into a porous medium. 


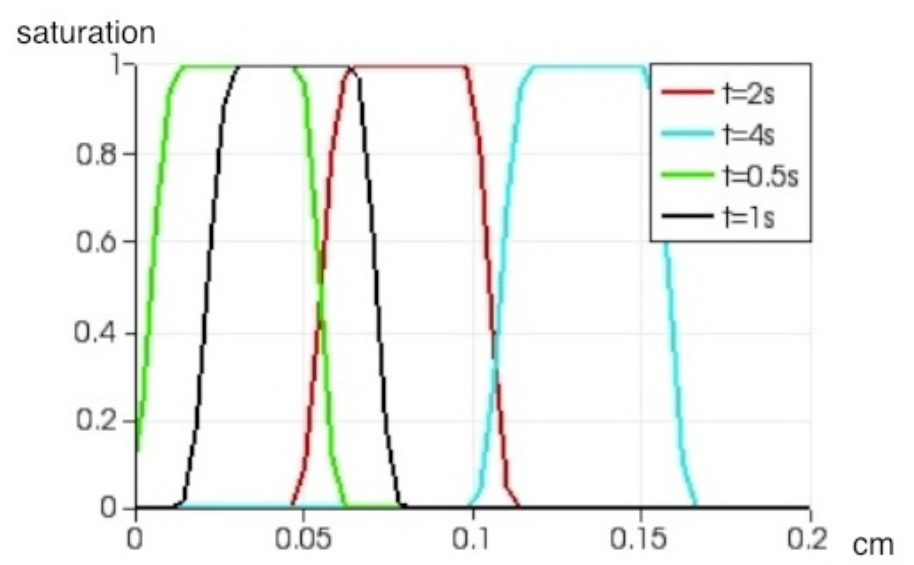

Figure 6: Simulated saturation levels for different times.

(2.13) Natural extensions of these simple simulations could be:

- Simulations using different geometries and several layers with different porosities.

- Add penetration of the decontaminant into the porous media.

- Add chemical reactions between the solid matrix and the fluids or between the two fluids.

OpenFOAM could be a useful tool for developing such simulations but requires better understanding of the software as well as the model equations considered here.

\section{Reaction and decontamination}

\subsection{Introduction}

(3.1) Most chemical agents are lipophilic (e.g. organophosphates), and as such are immiscible with water. Decontamination is usually performed by decanting an aqueous solution of cleanser on top of the contaminated region which, over time, seeps into the porous media and reacts with the adsorbed chemical agents. The product of the reaction is either soluble in water or in the contaminant, depending on the precise contaminant and the cleanser used. While the cleanser can be continuously applied on the top of the contaminated surface, little can be done to push the cleanser into the porous medium save for scrubbing and mopping. It is also difficult to experimentally track the progress of the reaction in the porous media. A key question that determines the efficacy of decontamination is the rate at which the contaminant can be annihilated. 
(3.2) To model the decontamination process, we consider two cases. In the first case, the product of the decontamination reaction is water soluble, and thus the contaminant layer is depleted as the reaction proceeds. In the second case, an oily product with similar density to the chemical agent is formed. Therefore, to first approximation, the total volume of the oil region does not change over time and the contaminant is continuously diluted with product as the reaction proceeds. In the models analysed in this section, we neglect the effects of hydrodynamics on the reaction - the finer aspects of multiphase porous medium flow will be considered later. However, as shown below, our simplified models of reaction already reveal some key physics that could underpin the optimisation of the decontamination process.

(3.3) Keeping the model general, we consider the generic decontamination reaction

$$
m C+q N \stackrel{k}{\longrightarrow} P
$$

where $C, N$ and $P$ denote cleanser, chemical agent and product, respectively, with $m$ and $q$ being the respective stoichiometric coefficients. The reaction rate is denoted by $k$ and is determined experimentally.

\subsection{Decontamination: water-soluble products}

(3.4) When the product is water-soluble, the aqueous phase of cleanser continuously "invades" the contaminant phase. To model that process, we consider a 1D free boundary problem of a diffusing cleanser with reactive boundary condition (see Fig. 7 for a schematic sketch of the set-up). Initially the aqueous cleanser layer occupies the region $-h<\hat{y}<0$. In the aqueous layer the cleanser concentration $\hat{c}$ satisfies the diffusion equation

$$
\frac{\partial \hat{c}}{\partial \hat{t}}=D_{c} \frac{\partial^{2} \hat{c}}{\partial \hat{y}^{2}}
$$

where $D_{c}$ is the diffusion coefficient of the cleanser in the aqueous solution. At the cleanser-contaminant boundary, which we denote as $\hat{y}=-\hat{s}(\hat{t})$, we have

$$
\frac{1}{m} D_{c} \frac{\partial \hat{c}}{\partial \hat{y}}(-\hat{s}(\hat{t}), \hat{t})=k \hat{c}(-\hat{s}(\hat{t}), \hat{t})^{m}
$$

and

$$
\frac{\mathrm{d} \hat{s}}{\mathrm{~d} \hat{t}}=\frac{q}{m} D_{c} V_{m} \frac{\partial \hat{c}}{\partial \hat{y}}(-\hat{s}(\hat{t}), \hat{t}) .
$$

Equation (5) is a consequence of chemistry - the left-hand side of Equation (5) the flux of cleanser at the interface, which equals the rate at which the reaction consumes the cleanser, and is described by the kinetic term on the right-hand side. Note that the contaminant concentration is constant and thus subsumed in the definition of $k$. Equation (6) is a statement of conservation of mass. The rate at which the interface moves, $\mathrm{d} \hat{s} / \mathrm{d} \hat{t}$, equals 
the volume of contaminant consumed per unit area per unit time, which is a product of the flux of contaminant $\frac{q}{m} D_{c} \partial \hat{c} / \partial \hat{y}$ evaluated at the moving interface, and the contaminant molecular volume $V_{m}$. At the top of the cleanser layer, we impose

$$
\hat{c}(0, \hat{t})=c_{0},
$$

as the cleanser is continuously replenished.

$$
\begin{array}{l|l|l}
\multicolumn{1}{c|}{y=0} & \multicolumn{1}{c}{c(0, t)=1} \\
\cline { 2 - 3 } y=-s(t) & \begin{array}{l}
c_{t}=c_{y y} \\
\text { aq. phase } \\
m C+q N \stackrel{k}{\rightarrow} P
\end{array} & c_{y}(-s, t)=K_{1} m c(-s, t)^{m} \\
\cline { 2 - 3 } & \begin{array}{l}
\text { contaminant } \\
\text { phase }
\end{array} & \\
& &
\end{array}
$$

Figure 7: Schematic sketch of the geometry and set-up. Dimensionless equations are used in the sketch and subscripts denote partial derivatives.

(3.5) Initially, the cleanser concentration is uniform and

$$
\hat{c}(\hat{y}, 0)=c_{0} .
$$

Thus the interface is located at $\hat{y}=-h$, hence

$$
\hat{s}(0)=h .
$$

(3.6) We non-dimensionalise equations (4)-(6) by letting

$$
\hat{t}=\left(h^{2} / D_{c}\right) t \quad(\hat{y}, \hat{s})=h(y, s), \quad \hat{c}=c_{0} c .
$$

Thus, we have

$$
\frac{\partial c}{\partial t}=\frac{\partial^{2} c}{\partial y^{2}}
$$

with free boundary conditions

$$
\frac{1}{m} \frac{\partial c}{\partial y}(-s(t), t)=K_{1} c(-s(t), t)^{m}, \quad \frac{\mathrm{d} s}{\mathrm{~d} t}=\frac{q}{m} B \frac{\partial c}{\partial y}(-s(t), t),
$$

and Dirichlet condition on the top

$$
c(0, t)=1 \text {. }
$$

The initial conditions read

$$
s(0)=1, \quad c(y, 0)=1 .
$$


This non-dimensionalisation identifies the key dimensionless parameters

$$
K_{1} \equiv \frac{k c_{0}^{m}}{\left(D_{c} c_{0}\right) / h}, B \equiv c_{0} V_{m}
$$

(3.7) Whilst the moving boundary problem defined in (11)-(14) can only be fully solved using numerics, it is possible to make some analytic progress. In particular, we can describe how the free-boundary moves at short and long times. We therefore split the analysis into these two cases, beginning with the short-time analysis.

\section{Short-time asymptotic analysis: $t \ll 1$}

(3.8) In order to formalise the analysis, we introduce the artificial small parameter $\epsilon \ll 1$ and define $T=t / \epsilon=O(1)$ to investigate the short-time behaviour. We deliberately avoid scaling $s$ until necessary, as we expect the correct scaling to become apparent from the analysis. We therefore take $s(t)=1+\delta s_{1}(T)$, where $\delta(\epsilon) \ll 1$ and is to be determined along with $s_{1}(T)$. This time scaling modifies the governing equation (11) to

$$
\frac{\partial c}{\partial T}=\epsilon \frac{\partial^{2} c}{\partial y^{2}}
$$

The form of (16) suggests that the cleanser concentration is constant (at a value of $c \equiv 1$, taken from the initial and boundary conditions) throughout most of the region, but that there is a sharp change in a small region that drives the boundary movement. We therefore zoom into a region close to the free boundary, taking $y=-s(t)+\epsilon^{1 / 2} Y$, for $Y>0$ and $Y=O(1)$. Then, the governing equation (16) becomes

$$
\frac{\partial c}{\partial T}=\frac{\partial^{2} c}{\partial Y^{2}}
$$

and the boundary conditions (12) (including the time scaling) become

$$
\frac{1}{m} \frac{\partial c}{\partial Y}(0, T)=\epsilon^{1 / 2} K_{1} c(0, T)^{m}, \quad \delta \frac{\mathrm{d} s_{1}}{\mathrm{~d} T}=\epsilon^{1 / 2} \frac{q}{m} B \frac{\partial c}{\partial Y}(0, T) .
$$

The first of these suggests no flux of cleanser through the boundary at leading order. As the concentration away from this boundary is $c=1$, we take the asymptotic form

$$
c \sim 1+\epsilon^{1 / 2} c_{1}(Y, T)+O(\epsilon)
$$

where $c_{1}$ satisfies the governing equation (17), and the free boundary conditions (18) become

$$
\frac{\partial c_{1}}{\partial Y}(0, T)=m K_{1}+O\left(\epsilon^{1 / 2}\right), \quad \delta \frac{\mathrm{d} s_{1}}{\mathrm{~d} T}=\epsilon \frac{q}{m} B \frac{\partial c_{1}}{\partial Y}(0, T),
$$

and the second of these gives the scaling $\delta=\epsilon$. 
(3.9) If we were interested in the value of the concentration, we could then solve the full equation for $c_{1}$. However, our interest lies in determining $s_{1}(T)$, which we can obtain via substitution of (20a) into (20b) to obtain

$$
\frac{\mathrm{d} s_{1}}{\mathrm{~d} T}=q B K_{1} .
$$

The right-hand side is a constant, and so this is easily integrated to obtain a linear function of time. In terms of the original variables $t$ and $s(t)$, the solution is then given by

$$
s(t) \sim 1+q B K_{1} t
$$

for $t \ll 1$.

\section{Long-time asymptotic analysis: $t \gg 1$}

(3.10) For the long-time analysis, it is instructive to work in a fixed domain. Hence, we make the transformation $\xi=y / s(t)$. The full problem (11)-(14) then becomes

$$
\begin{aligned}
s^{2} \frac{\partial c}{\partial t} & =\frac{\partial^{2} c}{\partial \xi^{2}}+\xi s \frac{\mathrm{d} s}{\mathrm{~d} t} \frac{\partial c}{\partial \xi}, & & \text { for } \xi \in(-1,0), \\
c & =1, & & \text { on } \xi=0, \\
\frac{\partial c}{\partial \xi} & =K_{1} m s c^{m}, \quad m s \frac{\mathrm{d} s}{\mathrm{~d} t}=q B \frac{\partial c}{\partial \xi}, & & \text { on } \xi=-1, \\
c & =1, \quad s=1, & & \text { at } t=0 .
\end{aligned}
$$

(3.11) Similarly to the short time analysis in (3.8), we introduce the artificial small parameter $\epsilon \ll 1$, but now define $T=\epsilon t=O(1)$ to investigate the longtime behaviour. The benefit of moving to a fixed domain is that, as we expect a diffusion-driven long-term behaviour over the entire domain, the time scaling immediately suggests a scaling of $S(T)=\epsilon^{1 / 2} s(t)$ for the free boundary. These scalings change (23) and (25) to

$$
\begin{aligned}
S^{2} \frac{\partial c}{\partial T} & =\frac{\partial^{2} c}{\partial \xi^{2}}+\xi S \frac{\mathrm{d} S}{\mathrm{~d} T} \frac{\partial c}{\partial \xi}, & & \text { for } \xi \in(-1,0), \\
\epsilon^{1 / 2} \frac{\partial c}{\partial \xi} & =K_{1} m S c^{m}, \quad m S \frac{\mathrm{d} S}{\mathrm{~d} T}=q B \frac{\partial c}{\partial \xi}, & & \text { on } \xi=-1 .
\end{aligned}
$$

We note that, at leading order, the cleanser uptake condition (28a) becomes a Dirichlet condition for $c$ on $\xi=-1$, and that the other equations are left unchanged. In fact, as we now have $c=0$ on $\xi=-1$, the invariant transformation $T \mapsto \nu^{2} T$ and $S \mapsto \nu S$ holds for the entire leading order problem. Therefore, we expect $S(T)=\lambda T^{1 / 2}$, where $\lambda$ is a constant to be determined. 
(3.12) Using the form of $S(T)$, the problem to solve is

$$
\begin{aligned}
\lambda^{2} T \frac{\partial c}{\partial T} & =\frac{\partial^{2} c}{\partial \xi^{2}}+\frac{\lambda^{2}}{2} \xi \frac{\partial c}{\partial \xi}, & & \text { for } \xi \in(-1,0), \\
c & =1, & & \text { on } \xi=0, \\
c & =0, \quad m \lambda^{2}=2 q B \frac{\partial c}{\partial \xi}, & & \text { on } \xi=-1 .
\end{aligned}
$$

The boundary conditions are independent of time, so if there is a stable time-independent solution to this problem, it is a solution to the full 'timedependent' problem. Equation (29) suggests that all linear perturbations in $\xi$ will decay exponentially in $T$, so any time-independent solution is stable. Therefore, we look for solutions of the form $c=c(\xi)$. The governing equation (29) then becomes

$$
0=\frac{\mathrm{d}^{2} c}{\mathrm{~d} \xi^{2}}+\frac{\lambda^{2}}{2} \xi \frac{\mathrm{d} c}{\mathrm{~d} \xi}
$$

which is solved by

$$
c(\xi)=1-\frac{\int_{\xi}^{0} \exp \left(-\lambda^{2} s^{2} / 4\right) \mathrm{d} s}{\int_{-1}^{0} \exp \left(-\lambda^{2} s^{2} / 4\right) \mathrm{d} s} .
$$

Substituting this into the equation for the boundary movement (31b) and rearranging, we obtain a transcendental equation for $\lambda$ as follows

$$
m \lambda^{2}=2 q B \frac{\exp \left(-\lambda^{2} / 4\right)}{\int_{0}^{1} \exp \left(-\lambda^{2} s^{2} / 4\right) \mathrm{d} s} .
$$

This can then be solved numerically for an arbitrary accuracy, and the long-time behaviour in the original variables is

$$
s(t) \sim \lambda t^{1 / 2}+o\left(t^{1 / 2}\right)
$$

where $\lambda$ satisfies (34).

\section{Numerical solution}

(3.13) To solve the free boundary problem numerically, we revert to scaled coordinates $y=y / s(t)$ (as detailed in $(23)-(26)$ ) and solve the resulting semidiscrete equation using central difference in space and the implicit MATLAB integrator ode15s to propagate in time. More specifically, denoting $\Delta y=1 / N$ as the grid spacing, $y_{i}=i \Delta y$ as the location of gridpoints and $c_{(i)}(t)$ as the numerical approximation of the solution at $y_{i}$, the semi-discrete system of equations reads

$$
\frac{\partial c_{(0)}}{\partial t}=1
$$




$$
\begin{gathered}
\frac{\partial c_{(j)}}{\partial t}=\frac{1}{s^{2}}\left[\frac{c_{(j-1)}-2 c_{(j)}+c_{(j+1)}}{\Delta y^{2}}+s \frac{\mathrm{d} s}{\mathrm{~d} t} j\left(c_{(j+1)}-c_{(j-1)}\right)\right], 2<j<N-1 \\
\frac{\partial c_{(N)}}{\partial t}=\frac{1}{s^{2}}\left[\frac{2 c_{(N-1)}-2 c_{(N)}-2 s K m c_{(N)}^{m}}{\Delta y^{2}}-s^{2} \frac{\mathrm{d} s}{\mathrm{~d} t} K m c_{(N)}^{m}\right]
\end{gathered}
$$

with

$$
\frac{\mathrm{d} s}{\mathrm{~d} t}=q K_{1} B c_{(N)}^{m}
$$

(3.14) The resulting solution for $m=q=1, K_{1}=10$ and $B=1$ is illustrated in Fig. 8. The initial linear regime (I) is reaction-limited, and the speed of interface motion is directly proportional to the rate of reaction $K_{1}$. At later time, the system transits into the depletion regime (II). The cleanser in the immediate vicinity of the interface is depleted, stalling the movement of the interface. The depletion regime continues until diffusion brings cleanser back near the vicinity of the interface, and reignites the movement of the interface (regime (III)). Finally, the movement of the interface comes into balance with mass-transport (regime (IV)) and the the interface position scales as the characteristic diffusion scaling $\sim t^{1 / 2}$ with the pre-factors independent of the reaction rate.

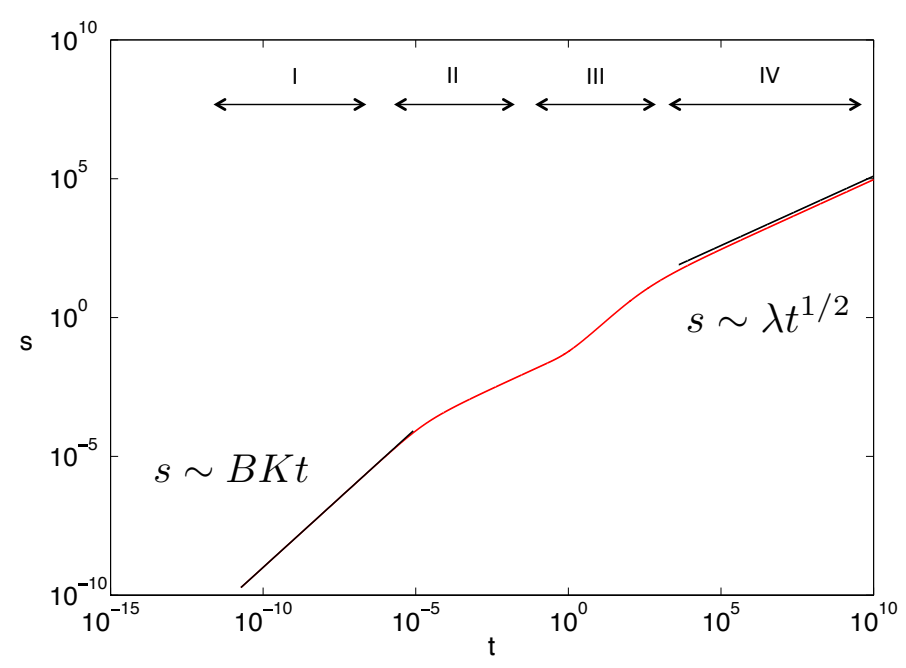

Figure 8: The numerical solutions (red curve) compares well with asymptotic analysis. The figure shows the case $m=q=1, K_{1}=10$ and $B=1$, and the arrows indicates approximate locations of the regimes as analysed in the main text. 


\subsection{Decontamination: oil-soluble products}

(3.15) In the case where the products are oil-soluble, the contaminant is continuously diluted as the reaction proceeds, and the overall volume of the contaminant layer is roughly constant. Thus the interface is not moving and we can model the process using two coupled diffusion equations for the cleanser concentration and contaminant concentration on a fixed domain. For notational simplicity we define $0<\hat{y}<h$ as the aqueous phase region and $-l<\hat{y}<0$ as the contaminant phase region. Our model reads

$$
\begin{gathered}
\frac{\partial \hat{c}}{\partial \hat{t}}=D_{c} \frac{\partial^{2} \hat{c}}{\partial \hat{y}^{2}}, \quad \hat{y} \in[0, h], \\
\frac{\partial \hat{n}}{\partial \hat{t}}=D_{n} \frac{\partial^{2} \hat{n}}{\partial \hat{y}^{2}}, \quad \hat{y} \in[-l, 0],
\end{gathered}
$$

with boundary conditions

$$
\begin{aligned}
\frac{1}{m} D_{c} \frac{\partial \hat{c}}{\partial \hat{y}}(0, \hat{t}) & =-k \hat{c}(0, \hat{t})^{m} \hat{n}(0, \hat{t})^{q} \\
\frac{1}{q} D_{n} \frac{\partial \hat{n}}{\partial \hat{y}}(0, \hat{t}) & =k \hat{c}(0, \hat{t})^{m} \hat{n}(0, \hat{t})^{q} \\
\hat{c}(h, \hat{t}) & =c_{0} \\
\frac{\partial \hat{n}}{\partial \hat{y}}(-l, \hat{t}) & =0 .
\end{aligned}
$$

The initial conditions are

$$
\begin{aligned}
& \hat{c}(\hat{y}, 0)=c_{0}, \\
& \hat{n}(\hat{y}, 0)=n_{0} .
\end{aligned}
$$

Non-dimensionalising length scales with respect to $h$, time scales with respect to $h^{2} / D_{c}$ and concentrations with respect to the initial concentrations, we arrive at

$$
\begin{gathered}
\frac{\partial c}{\partial t}=\frac{\partial^{2} c}{\partial y^{2}}, \quad y \in[0,1], \\
\frac{\partial n}{\partial t}=D \frac{\partial^{2} n}{\partial y^{2}}, \quad y \in[-\alpha, 0],
\end{gathered}
$$

with

$$
\begin{aligned}
\frac{1}{m} \frac{\partial c}{\partial y}(0, t) & =-K c(0, t)^{m} n(0, t)^{q} \\
\frac{1}{q} D \frac{\partial n}{\partial y}(0, t) & =K \frac{c_{0}}{n_{0}} c(0, t)^{m} n(0, t)^{q} \\
c(1, t) & =1 \\
\frac{\partial n}{\partial y}(-\alpha, t) & =0
\end{aligned}
$$


and initial conditions

$$
\begin{aligned}
& c(y, 0)=1, \\
& n(y, 0)=1 .
\end{aligned}
$$

The dimensionless parameters are

$$
D \equiv \frac{D_{n}}{D_{c}}, K_{2}=\frac{k c_{0}^{m} n_{0}^{q}}{\left(D_{c} c_{0}\right) / h}, \alpha=\frac{l}{h} .
$$

(3.16) Fig. 9 shows the numerical solution of Equations (48) and (49) with $c_{0}=n_{0}$, $K_{2}=10, \alpha=1$ and $m=q=1$ using the MATLAB routine pdepe. The figure shows that the short time behaviour is characterised by the depletion of cleanser near the interface, before diffusion of cleanser from the bulk replenishes it. This diffusion-driven process only asymptotically removes the contaminant - this is because the contaminant concentration is continuously diluted as the reaction proceeds.
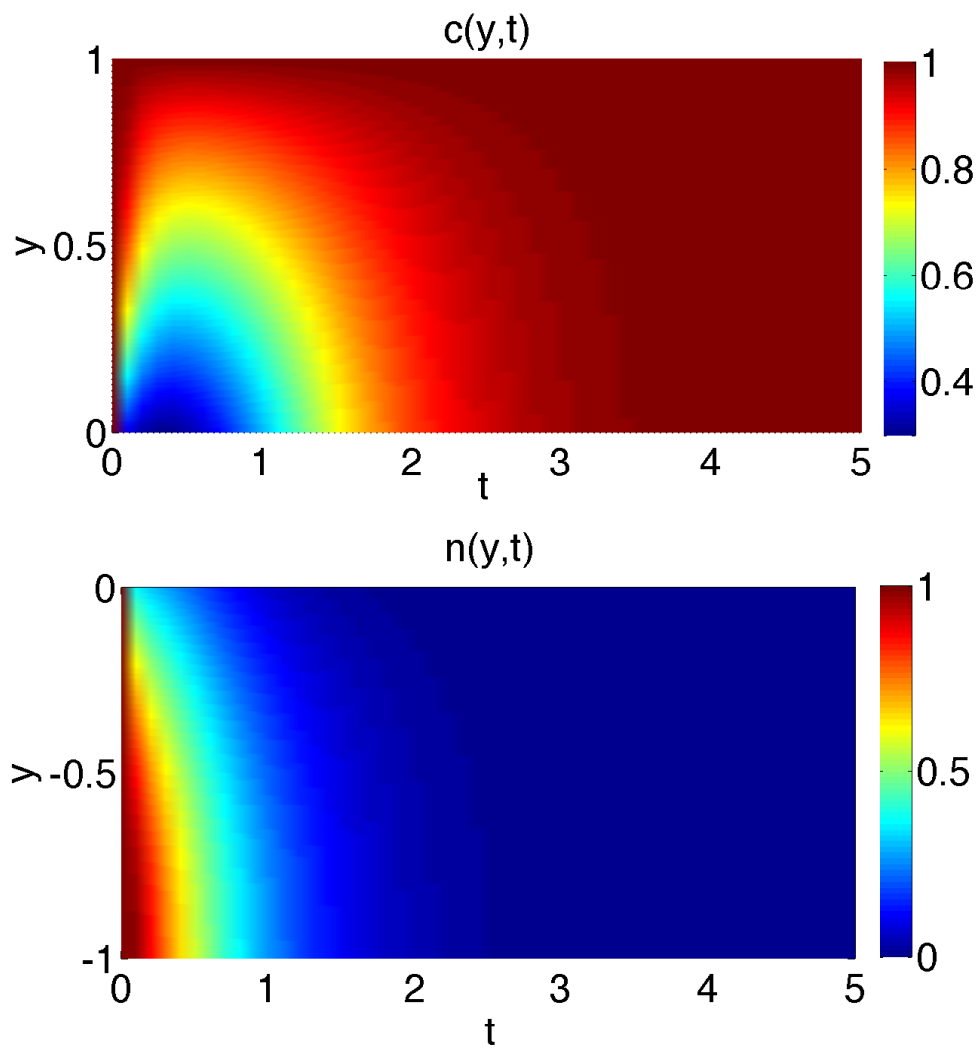

Figure 9: Numerical solution of Equations (48) and (49) with $c_{0}=$ $n_{0}, K_{2}=10, \alpha=1$ and $m=q=1$. The plots show the initial depletion of cleanser near the interface before mass transport from the bulk replenishes it. 
(3.17) A quantity of practical interest for decontamination is the total amount of contaminant $n_{\text {tot }}$ left in the system as a function of time, which is given by

$$
n_{t o t}(t)=\int_{-\alpha}^{0} n(y, t) \mathrm{d} y .
$$

Fig. 10 shows the qualitative dependence of $n_{\text {tot }}$ on the reaction kinetics. Expectedly, decontamination proceeds at a slower rate if more than one molecule of decontaminant is required to annihilate a contaminant molecule. Intuitively, one would expect the converse to hold as well, i.e. the greater the number of contaminant molecules that a molecule of decontaminant can remove, the more effective the decontamination process is. However, the contrary is shown in Fig. 10 - despite a larger initial drop in $n_{t o t}$, over time, the $m=1, q=2$ case is significantly less effective than the $m=1, q=1$ case. The trimolecular kinetics creates a much larger concentration drop in contaminant concentration and this "depletion" zone penetrates deeper into the contaminant layer. Therefore, it is more difficult for diffusive mass transport to bring contaminant to the interface and continue the reaction. For the same reason, the $m=2, q=2$ case is inferior to $m=1 q=1$ case.

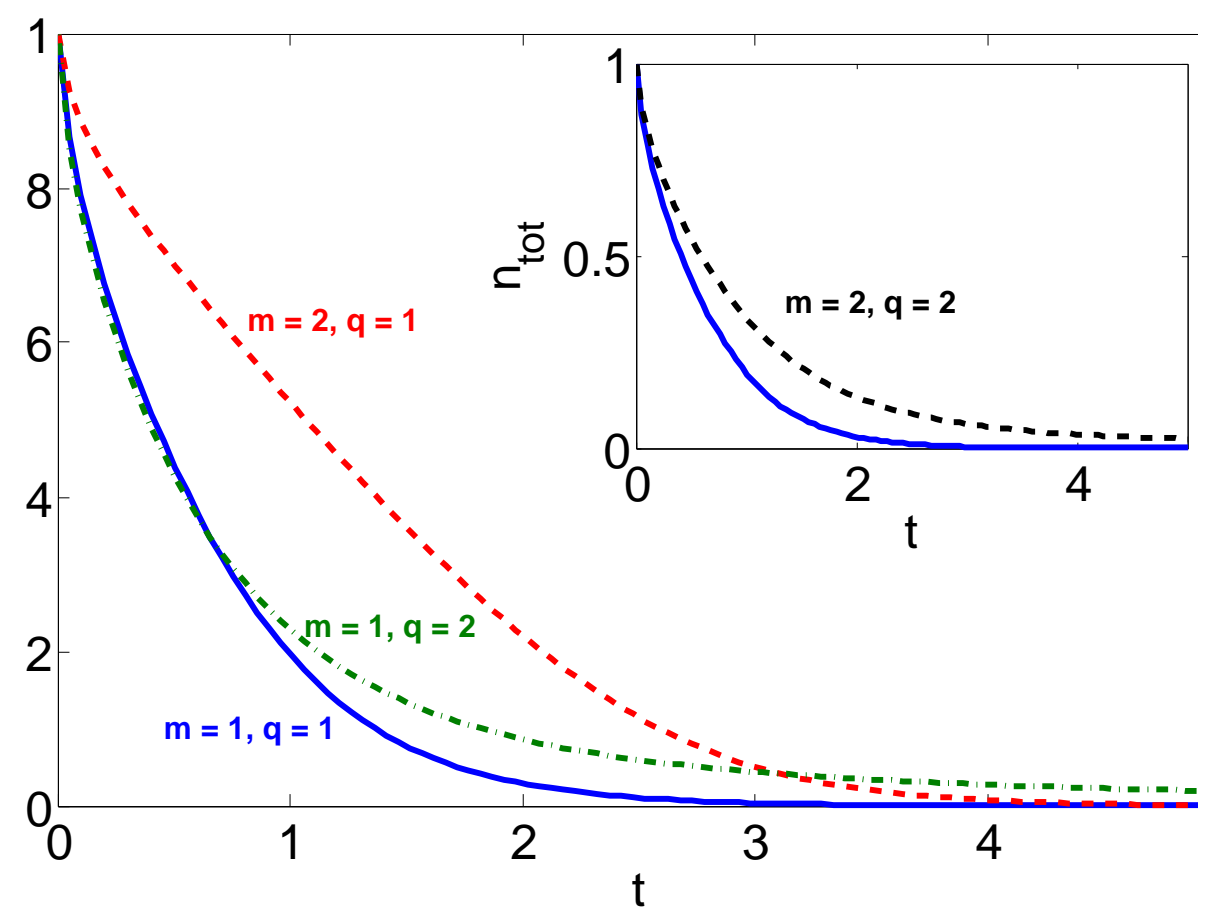

Figure 10: The integrated total amount of contaminant $n_{t o t}$ as a function of time plotted for different molecularity of the decontamination reaction. Other parameters are the same as Fig. 9

(3.18) The effect of diffusion coefficient is examined in Fig. 11. For contaminant with low self diffusion coefficient (or large viscosity), decontamination via 
this approach is particularly ineffective. Slow mass transport of contaminant from the bulk to the reacting interface greatly hinders the decontamination reaction.

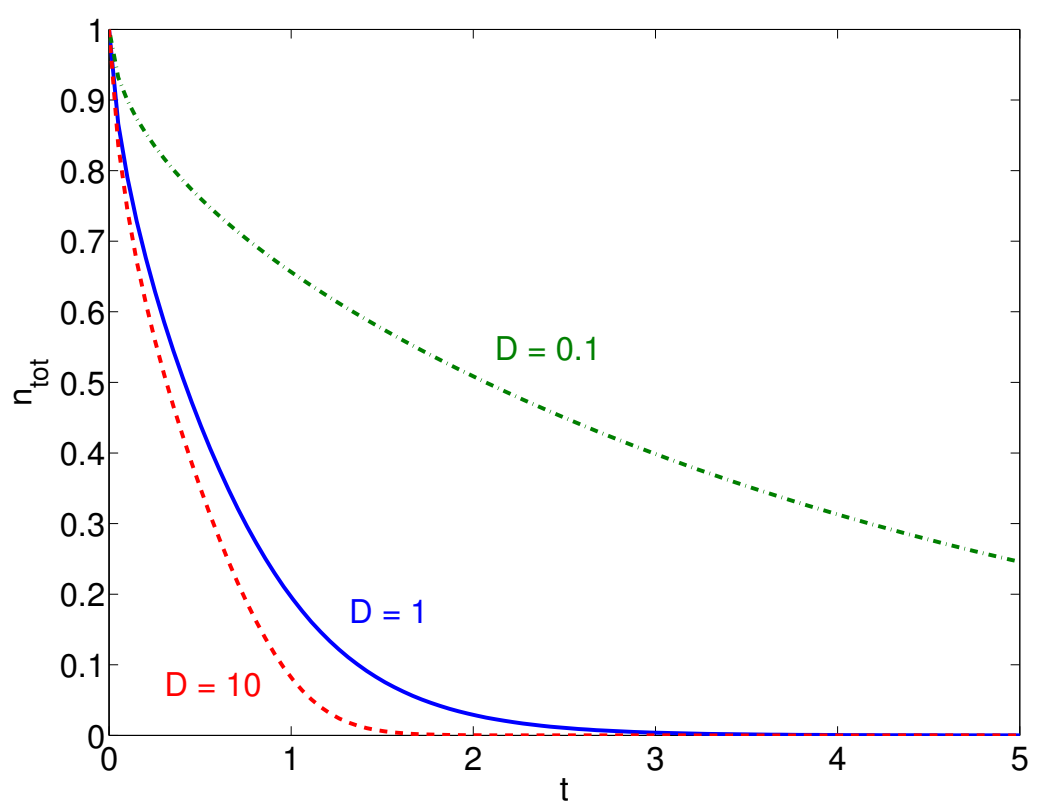

Figure 11: The integrated total amount of contaminant $n_{\text {tot }}$ as a function of time plotted for different diffusion coefficient. As the timescale is non-dimensionalised with respect to the cleanser diffusion coefficient $D_{c}$, the plot can be interpreted as varying the contaminant self-diffusion coefficient $D_{n}$ whilst keeping other parameters fixed.

\subsection{Conclusion}

(3.19) Our models show that decontamination is most effective when the product dissolves in the aqueous phase rather than the contaminant phase. A product that is miscible with the contaminant will continuously dilute the contaminant as the reaction proceeds, and the reaction is only complete as $t \rightarrow \infty$. This is particularly problematic for viscous contaminant with low diffusion coefficient. On the other hand, a water-soluble product will drive a "reaction front" that annihilate the contaminant effectively in finite time with the rate of decontamination independent of the contaminant viscosity.

(3.20) If there are no alternatives but to use a reaction that produces oil-soluble product, our calculations suggest that a bimolecular decontamination reaction is optimal, and reactions with higher molecularities will slow down the decontamination process. 


\section{A two-dimensional flow and reaction model for decontamination}

\subsection{Model overview and governing equations}

(4.1) In this section we introduce fluid flow into our system. We model the flow of a "cleanser" and a "nasty" fluid through a porous medium (such as a carpet or concrete) which has a finite thickness and lies on top of a relatively impermeable surface (Fig. 12). We assume that the cleanser is constantly replenished at the top and we model scrubbing of the upper boundary by imposing a variable pressure there. The cleanser is considered to be an aqueous solution of a neutralising agent and the products of the reaction between "cleanser" and "nasty" dissolve in the former, therefore only the concentration of the cleanser needs to be considered. Since the cleanser is continually replenished above the surface, we assume its concentration at ground level to be constant. We take the interface between the two phases to be sharp and the reaction as instantaneous. Since the width of a typical spill of a hazardous material is much larger than its depth we consider the two fluids to be infinite in the horizontal direction and ignore edge effects.

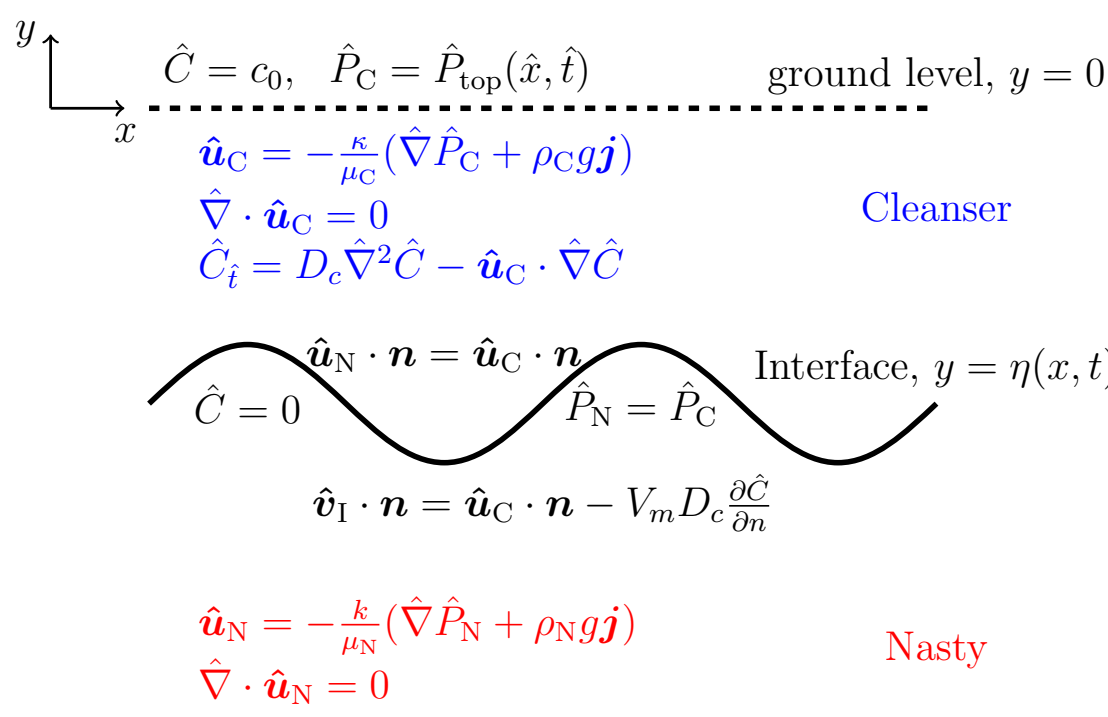

$$
\begin{array}{ll}
\boldsymbol{u}_{\mathrm{N}} \cdot \boldsymbol{j}=0 & \text { rigid boundary, } y=-H
\end{array}
$$

Figure 12: Overview of the equations for the two-dimensional flow and reaction model.

(4.2) As in $\S 2.2$, we use Darcy's equations to describe flow through the porous medium. The fluid velocities $\hat{\boldsymbol{u}}_{\mathrm{C}}, \hat{\boldsymbol{u}}_{\mathrm{N}}$, and the pressures $\hat{P}_{\mathrm{C}}, \hat{P}_{\mathrm{N}}$, of the 
"cleanser" and the "nasty" phases respectively, are related via the following equations:

$$
\begin{array}{ll}
\hat{\boldsymbol{u}}_{\mathrm{C}}=-\frac{\kappa}{\mu_{\mathrm{C}}}\left(\hat{\nabla} \hat{P}_{\mathrm{C}}+\rho_{\mathrm{C}} g \boldsymbol{j}\right), & \hat{\nabla} \cdot \hat{\boldsymbol{u}}_{\mathrm{C}}=0 \\
\hat{\boldsymbol{u}}_{\mathrm{N}}=-\frac{\kappa}{\mu_{\mathrm{N}}}\left(\hat{\nabla} \hat{P}_{\mathrm{N}}+\rho_{\mathrm{N}} g \boldsymbol{j}\right), & \hat{\nabla} \cdot \hat{\boldsymbol{u}}_{\mathrm{N}}=0
\end{array}
$$

where $\kappa$ is the permeability of the porous material, $\mu_{\mathrm{C}}, \mu_{\mathrm{N}}$ are the viscosities, $\rho_{\mathrm{C}}, \rho_{\mathrm{N}}$ are the densities of the two phases and $-g \boldsymbol{j}$ is the gravitational acceleration. Eliminating the fluid velocities, we obtain a Laplacian for the pressure in both equations:

$$
\hat{\nabla}^{2} \hat{P}_{\mathrm{C}}=0, \quad \hat{\nabla}^{2} \hat{P}_{\mathrm{N}}=0 .
$$

The cleanser concentration is given by an advection-diffusion equation:

$$
\frac{\partial \hat{C}}{\partial \hat{t}}=D_{c} \hat{\nabla}^{2} \hat{C}-\hat{\boldsymbol{u}}_{\mathrm{C}} \cdot \hat{\nabla} \hat{C}
$$

which again can be written in terms of the pressure instead of the fluid velocity:

$$
\frac{\partial \hat{C}}{\partial \hat{t}}=D_{c} \hat{\nabla}^{2} \hat{C}+\frac{\kappa}{\mu_{\mathrm{C}}}\left(\hat{\nabla} \hat{P}_{\mathrm{C}}+\rho_{\mathrm{C}} g \boldsymbol{j}\right) \cdot \hat{\nabla} \hat{C} .
$$

(4.3) In general, the interface between the two phases is given by a parameterization $\left(\hat{x}_{\mathrm{I}}(\hat{s}, \hat{t}), \hat{y}_{\mathrm{I}}(\hat{s}, \hat{t})\right)$. We assume that this parameterization satisfies

$$
\hat{y}_{\mathrm{I}}(\hat{s}, \hat{t})=\eta\left(\hat{x}_{\mathrm{I}}(\hat{s}, \hat{t}), \hat{t}\right)
$$

for some function $\eta(\hat{x}, \hat{t})$, such that

$$
\frac{\partial \hat{y}_{\mathrm{I}}}{\partial \hat{s}}=\frac{\partial \eta}{\partial \hat{x}} \frac{\partial \hat{x}_{\mathrm{I}}}{\partial \hat{s}}, \quad \frac{\partial \hat{y}_{\mathrm{I}}}{\partial \hat{t}}=\frac{\partial \eta}{\partial \hat{x}} \frac{\partial \hat{x}_{\mathrm{I}}}{\partial \hat{t}}+\frac{\partial \eta}{\partial \hat{t}}
$$

and we thus obtain the (upward) normal to the interface as

$$
\begin{aligned}
\boldsymbol{n} & =\left(\left(\frac{\partial \hat{x}_{\mathrm{I}}}{\partial \hat{s}}\right)^{2}+\left(\frac{\partial \hat{y}_{\mathrm{I}}}{\partial \hat{s}}\right)^{2}\right)^{-\frac{1}{2}}\left(-\frac{\partial \hat{y}_{\mathrm{I}}}{\partial \hat{s}}, \frac{\partial \hat{x}_{\mathrm{I}}}{\partial \hat{s}}\right) \\
& =\left(1+\left(\frac{\partial \eta}{\partial \hat{x}}\right)^{2}\right)^{-\frac{1}{2}}\left(-\frac{\partial \eta}{\partial \hat{x}}, 1\right)
\end{aligned}
$$

and the normal interface velocity as

$$
\hat{\boldsymbol{v}}_{\mathrm{I}} \cdot \boldsymbol{n}=\left(\frac{\partial \hat{x}_{\mathrm{I}}}{\partial \hat{t}}, \frac{\partial \hat{y}_{\mathrm{I}}}{\partial \hat{t}}\right) \cdot \boldsymbol{n}=\left(1+\left(\frac{\partial \eta}{\partial \hat{x}}\right)^{2}\right)^{-\frac{1}{2}} \frac{\partial \eta}{\partial \hat{t}} .
$$


The interface is driven by both the flow in the two phases and by "nasty" fluid being used up due to the reaction with the cleaning agent (compare $\S 3.2$ ). This reaction is limited by the diffusion of cleaning agent into the interface:

$$
\hat{\boldsymbol{v}}_{\mathrm{I}} \cdot \boldsymbol{n}=\hat{\boldsymbol{u}}_{\mathrm{C}} \cdot \boldsymbol{n}-V_{m} D_{c} \hat{\nabla} \hat{C} \cdot \boldsymbol{n},
$$

recalling from $\S 3.2$ that $V_{m}$ is the molar volume of contaminant, and allows us to relate the flux of cleanser into the interface to the interfacial speed. These last two equations can be combined with the Darcy flow equation to obtain an interface evolution equation in terms of the pressure and the cleaning agent diffusion speed:

$$
\begin{aligned}
\frac{\partial \eta}{\partial \hat{t}} & =-\left(\frac{\kappa}{\mu_{\mathrm{C}}} \hat{\nabla} \hat{P}_{\mathrm{C}}+\frac{\kappa \rho_{\mathrm{C}} g}{\mu_{\mathrm{C}}} \boldsymbol{j}+V_{m} D_{c} \hat{\nabla} \hat{C}\right) \cdot\left(-\frac{\partial \eta}{\partial \hat{x}}, 1\right) \cdot \\
& =\frac{\partial \eta}{\partial \hat{x}}\left(\frac{\kappa}{\mu_{\mathrm{C}}} \frac{\partial \hat{P}_{\mathrm{C}}}{\partial \hat{x}}+V_{m} D_{c} \frac{\partial \hat{C}}{\partial \hat{x}}\right)-\frac{\kappa}{\mu_{\mathrm{C}}} \frac{\partial \hat{P}_{\mathrm{C}}}{\partial \hat{y}}-\frac{\kappa \rho_{\mathrm{C}} g}{\mu_{\mathrm{C}}}-V_{m} D_{c} \frac{\partial \hat{C}}{\partial \hat{y}}
\end{aligned}
$$

(4.4) Since the reaction is instantaneous, any cleaning agent that reaches the interface is consumed immediately so the concentration at the interface has to be zero:

$$
\hat{C}=0 \quad \text { at } \hat{y}=\eta(\hat{x}, \hat{t}) .
$$

Furthermore, we require the pressure to be continuous across the interface:

$$
\hat{P}_{\mathrm{C}}=\hat{P}_{\mathrm{N}} \quad \text { at } \hat{y}=\eta(\hat{x}, \hat{t}) .
$$

Finally, the normal velocities of the two fluids have to be continuous at the interface to prevent the two phases to overlap or detach. This gives us a condition on the pressure gradient:

$$
\left(\hat{\nabla} \hat{P}_{\mathrm{C}}-\frac{\mu_{\mathrm{C}}}{\mu_{\mathrm{N}}} \hat{\nabla} \hat{P}_{\mathrm{N}}\right) \cdot \boldsymbol{n}=\left(\frac{\mu_{\mathrm{C}}}{\mu_{\mathrm{N}}} \rho_{\mathrm{N}}-\rho_{\mathrm{C}}\right) g \boldsymbol{j} \cdot \boldsymbol{n} \quad \text { at } \hat{y}=\eta(\hat{x}, \hat{t}) .
$$

(4.5) Since we've assumed that the cleanser is constantly replenished at the upper boundary, we take the concentration of $\hat{C}$ to be the constant value of $c_{0}$ at the top. In order to model the scrubbing we apply a pressure $\hat{P}_{\text {top }}$ which depends on $\hat{x}$ and $\hat{t}$. We assumed that the porous medium lies on top of an impermeable medium, hence we have no slip at the lower boundary. The boundary conditions then are:

$$
\begin{aligned}
\hat{C} & =c_{0} & & \text { at } \hat{y}=0, \\
\hat{P}_{\mathrm{C}} & =\hat{P}_{\text {top }}(\hat{x}, \hat{t}) & & \text { at } \hat{y}=0, \\
\frac{\partial \hat{P}_{\mathrm{N}}}{\partial \hat{y}} & =-\rho_{\mathrm{N}} g & & \text { at } \hat{y}=-H .
\end{aligned}
$$

(4.6) To summarise, we need to solve Laplace's equation for the pressure in both phases (60), the advection-diffusion equation for the cleanser concentration (62) and the evolution equation for the interface (68) together with the boundary conditions (69)-(74). 


\subsection{Non-dimensionalisation}

(4.7) To non-dimensionalise the problem we use the cleanser as the reference fluid. The characteristic scales are:

$$
\begin{aligned}
& x=\frac{\hat{x}}{L}, \\
& y=\frac{\hat{y}}{H}, \\
& t=\frac{H^{2}}{D_{c}} \hat{t}, \\
& P_{C}=\rho_{C} g H \hat{P}_{C}, \\
& P_{N}=\rho_{C} g H \hat{P}_{N}, \\
& C=c_{0} \hat{C} \text {. }
\end{aligned}
$$

The following dimensionless parameters emerge:

$$
\mathbb{A}=\frac{\rho_{N}}{\rho_{C}}, \quad M=\frac{\mu_{C}}{\mu_{N}}, \quad B=V_{m} c_{0}, \quad \mathbb{J}=\frac{\rho_{C} g H \kappa}{\mu_{C} D_{c}},
$$

which are the density and viscosity ratios, Damköhler number (equal to $B$ in (15)) and Péclet number respectively. The Damköhler number relates the reaction timescale to the convection timescale and gives an idea of the degree of conversion that can take place. The Péclet number relates the rate of advection to the rate of diffusion and gives an idea of the principle mechanism of how the concentration of the cleanser is transported. ${ }^{1}$

(4.8) The governing dimensionless equations for the pressure field are:

$$
\begin{aligned}
& \delta^{2} \frac{\partial^{2} P_{C}}{\partial x^{2}}+\frac{\partial^{2} P_{C}}{\partial y^{2}}=0 \\
& \delta^{2} \frac{\partial^{2} P_{N}}{\partial x^{2}}+\frac{\partial^{2} P_{N}}{\partial y^{2}}=0
\end{aligned}
$$

and the governing dimensionless equations for the cleanser concentration and for the interface are:

$$
\begin{aligned}
& \frac{\partial C}{\partial t}=\mathbb{J}\left(\delta^{2} \frac{\partial P_{C}}{\partial x} \frac{\partial C}{\partial x}+\frac{\partial P_{C}}{\partial y} \frac{\partial C}{\partial y}+\frac{\partial C}{\partial y}\right)+\delta^{2} \frac{\partial^{2} C}{\partial x^{2}}+\frac{\partial^{2} C}{\partial y^{2}} \\
& \frac{\partial \eta}{\partial t}=\delta^{2} \frac{\partial \eta}{\partial x}\left(\mathbb{J} \frac{\partial P_{C}}{\partial x}+B \frac{\partial C}{\partial x}\right)-\mathbb{J}\left(\frac{\partial P_{C}}{\partial y}+1\right)-B \frac{\partial C}{\partial y}
\end{aligned}
$$

The dimensionless boundary conditions are:

$$
\begin{aligned}
P_{C} & =P_{\text {top }}(x, t) \text { at } y=0, \\
\frac{\partial P_{N}}{\partial y} & =-\mathbb{A} \text { at } y=-1,
\end{aligned}
$$

and the matching conditions at the interface $y=\eta(x, t)$ are:

$$
\begin{aligned}
P_{C}-P_{N} & =0 \\
\delta^{2} \frac{\partial \eta}{\partial x}\left(\frac{\partial P_{C}}{\partial x}-M \frac{\partial P_{N}}{\partial x}\right)-\left(\frac{\partial P_{C}}{\partial y}-M \frac{\partial P_{N}}{\partial y}\right)-(1-\mathbb{A} M) & =0 .
\end{aligned}
$$

\footnotetext{
${ }^{1}$ Our definition of the Péclet number is non-standard, since the fluid velocity is governed by Darcy's law (59).
} 


\subsection{Long-wave asymptotics}

(4.9) In this subsection we consider the horizontal length scale of the porous medium to be much larger than the vertical length scale, i.e. $\delta^{2} \ll 1$. This corresponds to the physical case of a shallow porous substrate (e.g. carpet) on top of a relatively impermeable surface.

(4.10) We begin by expanding the solution in powers of $\delta^{2}$ :

$$
\begin{aligned}
P_{C}(x, y, t) & =P_{C 0}(x, y, t)+\delta^{2} P_{C 1}(x, y, t)+\mathcal{O}\left(\delta^{4}\right), \\
P_{N}(x, y, t) & =P_{N 0}(x, y, t)+\delta^{2} P_{N 1}(x, y, t)+\mathcal{O}\left(\delta^{4}\right), \\
C(x, y, t) & =C_{0}(x, y, t)+\delta^{2} C_{1}(x, y, t)+\mathcal{O}\left(\delta^{4}\right), \\
\eta(x, t) & =\eta_{0}(x, t)+\delta^{2} \eta_{1}(x, t)+\mathcal{O}\left(\delta^{4}\right) .
\end{aligned}
$$

The next step is to determine the governing equations at different orders of $\delta^{2}$ by substituting the above expressions (83)-(86) into the governing equations (75)-(78) and into the interfacial conditions (79)-(82).

(4.11) Substitution of the asymptotic expansions into the governing equations and into the boundary conditions is relatively straight forward. Particular care must be taken for the interfacial conditions since these are to be evaluated at the interface location $y=\eta_{0}(x, t)+\delta^{2} \eta_{1}(x, t)+\mathcal{O}\left(\delta^{4}\right)$. In order to derive the correct conditions at each order we must Taylor expand our unknowns about $y=\eta_{0}(x, t)$. For example, the Taylor expansion of $P_{N}$ about $y=\eta_{0}(x, t)$ is

$$
\begin{aligned}
& P_{N}(y=\eta(x, t), x, t) \\
& =P_{N 0}\left(y=\eta_{0}(x, t)+\delta^{2} \eta_{1}(x, t)+\ldots, x, t\right) \\
& \quad+\delta^{2} P_{N 1}\left(y=\eta_{0}(x, t)+\delta^{2} \eta_{1}(x, t)+\ldots, x, t\right)+\mathcal{O}\left(\delta^{4}\right) \\
& =\left.P_{N 0}\right|_{y=\eta_{0}}+\delta^{2}\left(\left.\eta_{1} \frac{\partial P_{N 0}}{\partial y}\right|_{y=\eta_{0}}+\left.P_{N 1}\right|_{y=\eta_{0}}\right)+\mathcal{O}\left(\delta^{4}\right) .
\end{aligned}
$$

\section{The $\mathcal{O}(1)$ matching conditions}

(4.12) The continuity of pressure condition at $\mathcal{O}(1)$ is:

$$
P_{C 0}=P_{N 0} \text { at } y=\eta_{0}(x, t)
$$

and at $\mathcal{O}\left(\delta^{2}\right)$ the condition is:

$$
\eta_{1} \frac{\partial P_{C 0}}{\partial y}+P_{C 1}=\eta_{1} \frac{\partial P_{N 0}}{\partial y}+P_{N 1} \text { at } y=\eta_{0}(x, t) .
$$

(4.13) The continuity of the velocity condition at $\mathcal{O}(1)$ is:

$$
-\frac{\partial P_{C 0}}{\partial y}+M \frac{\partial P_{N 0}}{\partial y}=1-\mathbb{A} M
$$


and at $\mathcal{O}\left(\delta^{2}\right)$ the condition is:

$$
\frac{\partial \eta_{0}}{\partial x}\left(\frac{\partial P_{C 0}}{\partial x}-M \frac{\partial P_{N 0}}{\partial x}\right)-\left(\eta_{1} \frac{\partial P_{C 0}}{\partial y}+P_{C 1}\right)+M\left(\eta_{1} \frac{\partial P_{N 0}}{\partial y}+P_{N 1}\right)=0
$$

\section{The $\mathcal{O}(1)$ equations}

(4.14) The leading order equations for the pressure are:

$$
\begin{aligned}
& \frac{\partial^{2} P_{C 0}}{\partial y^{2}}=0, \\
& \frac{\partial^{2} P_{N 0}}{\partial y^{2}}=0,
\end{aligned}
$$

which are subject to the conditions:

$$
\begin{aligned}
P_{C 0} & =P_{\text {top }} \text { at } y=0, \\
\frac{\partial P_{N 0}}{\partial y} & =-\mathbb{A} \text { at } y=1, \\
P_{C 0} & =P_{N 0} \text { at } y=\eta_{0}(x, t), \\
-\frac{\partial P_{C 0}}{\partial y}+M \frac{\partial P_{N 0}}{\partial y} & =1-\mathbb{A} M \text { at } y=\eta_{0}(x, t) .
\end{aligned}
$$

(4.15) Equations (92)-(97) can be solved to find:

$$
\begin{aligned}
& P_{C 0}=-y+P_{\text {top }}(x, t), \\
& P_{N 0}=-\mathbb{A} y+(\mathbb{A}-1) \eta(x, t)+P_{\text {top }}(x, t) .
\end{aligned}
$$

The leading order equations for the concentration of the cleanser and for the evolution of the interface are:

$$
\begin{aligned}
\frac{\partial C_{0}}{\partial t} & =\mathbb{J}\left(\frac{\partial P_{C 0}}{\partial y} \frac{\partial C_{0}}{\partial y}+\frac{\partial C_{0}}{\partial y}\right)+\frac{\partial^{2} C_{0}}{\partial y^{2}} \\
\frac{\partial \eta_{0}}{\partial t} & =-\mathbb{J}\left(\frac{\partial P_{C 0}}{\partial y}+1\right)-B \frac{\partial C_{0}}{\partial y}
\end{aligned}
$$

Substituting the expression for $P_{C 0}$ into (100)-(101) we find that the leading order governing equations reduce to the following Stefan problem:

$$
\begin{aligned}
\frac{\partial C_{0}}{\partial t} & =\frac{\partial^{2} C_{0}}{\partial y^{2}}, \\
\frac{\partial \eta_{0}}{\partial t} & =-B \frac{\partial C_{0}}{\partial y}, \\
\eta_{0}(x, t=0) & =\bar{\eta}(x), \\
C_{0} & =1 \text { at } y=0, \\
C_{0} & =0 \text { at } y=\eta_{0}(x, t),
\end{aligned}
$$




\subsection{Outlook}

(4.16) We have combined fluid flow and a reaction at the interface to derive a two-dimensional model for the decontamination process. We considered the long wavelength approximation in order to get a one-dimensional model applicable when considering a shallow porous substrate. At leading order, the equations reduce to a Stefan problem and can be compared to the equations derived in $\S 3.2$ : the only difference is in the treatment of the chemical concentration at the interface. The model in $\S 3.2$ takes into account the reaction rate whilst in this section the reaction is taken to be instantaneous.

(4.17) It is possible to derive the governing equations at the next order, taking particular care in Taylor expanding the matching conditions at the interface location. The algebra is cumbersome and the use of an algebra package such as Maple is recommended. We anticipate that the next order equations will incorporate hydrodynamic effects which would illuminate any possible mechanisms to enhance the decontamination process through instabilities such as fingering.

(4.18) For a numerical simulation, the main complication is that the interface needs to be tracked. Methods are available for kinematic fluid-fluid interfaces and it may well be possible to adapt those to include a reaction term. The long wavelength asymptotic approximation provides an analytic solution to compare and validate any two-dimensional numerical simulations conducted.

\section{Conclusions and outlook}

(5.1) Working with representatives from the UK Government Decontamination Service, the study group constructed mathematical models which addressed some of the pressing issues the Service faces when deciding which action to take upon release of a hazardous material.

(5.2) The primary concern is to determine how far a particular contaminant has spread into a substrate. First, we considered a one-dimensional problem in which a uniform layer of fluid seeps into an initially dry porous substrate. This process was modelled using Darcy's law of flow through a porous medium where we assumed in the first instance a sharp fluid interface in the porous medium. At the next level of complication we employed a diffuse interface model based on Richards' equation, since in reality we expect that there is a saturation gradient between the fully saturated and unsaturated regions in the porous material. The two models were in good agreement for early times, while at later times the diffuse interface model predicts the more realistic scenario in which the contaminant seeps deeper into the substrate even in the absence of further contaminant being supplied at the surface. This is in contrast to the model based on Darcy's law which 
predicts that the contaminant reaches finite depth after all the surface material has been used up. We therefore recommend that a diffuse interface model is used when approximating the depth reached by a contaminant in a porous material.

(5.3) The GDS were also concerned about the neutralisation process of a contaminant by the application of a decontaminant at the surface. The study group sought to determine whether it is possible to neutralise the full depth of the remaining toxic chemical after an initial surface clean-up has taken place. To understand this process, we considered two cases. The first case is one in which the product of the decontamination reaction is water soluble, and so the contaminant layer is used up as the reaction proceeds. We modelled this process by considering a one-dimensional free boundary problem of a diffusing cleanser with a reactive boundary condition. In the second case the reaction product enters the contaminant phase, so that the contaminant is continuously diluted as the reaction proceeds, and the overall volume of the contaminant layer is approximately constant. Therefore, the interface is not moving, allowing us to model the process using two coupled diffusion equations for the decontaminant concentration and contaminant concentration on a fixed domain. Using a mixture of asymptotic and numerical techniques we find that decontamination is most effective when the product is water soluble, rather than dissolving in the contaminant phase. This is due to the fact that in the latter case, the contaminant is continuously diluted and hence the reaction does not finish in finite time. In contrast, a water soluble product drives a "reaction front" that will neutralise remaining contaminant in the substrate in finite time. In the worst case scenario where only a reaction which produces a contaminant soluble product is available, we find that a bimolecular decontamination reaction is optimal.

(5.4) Finally, we attempted to incorporate the effects of hydrodynamics on the interface, by combining kinematic and reaction effects in an interface evolution condition. We find that in the long wavelength limit the governing equations reduce to those of a one-dimensional Stefan problem which can be compared to the equations derived earlier. More detailed approximate solutions, i.e., higher order corrections or numerical simulations were beyond the scope of the study group but pose interesting challenges for future research.

(5.5) We suggest that some interesting problems for future study groups to consider would be the following. First, to consider the scenario in which the contaminant leaches back out of the porous substrate due to density variations between the contaminant and decontaminant. Secondly, to determine whether it is possible to construct a diffuse interface model for the reaction process. There is also a possibility of so-called "viscous fingering" of the fluid interface to occur due to viscosity variations between phases. This would require a finer stability analysis of the governing equations, and it 
would yield a more intimate understanding of the depth by which the contaminant has been absorbed into the substrate. Finally, we suggest that a two-dimensional model could be constructed which incorporates both hydrodynamics and reaction effects, however this might prompt a numerical investigation and so expertise in appropriate scientific software packages would be required.

\section{Bibliography}

[1] Simon Parker and James Nally. Liquid interactions with porous media and the fate of toxic materials. Report from the European Study Group (ESGI) 85, available at http://www.cimr.uea.ac.uk/workshops/esgi85/ liquid-interactions/, 2012.

[2] James Acton, Herbert E. Huppert, and M. Grae Worster. Two-dimensional viscous gravity currents flowing over a deep porous medium. Journal of Fluid Mechanics, 440:359-380, 2001.

[3] OpenFOAM: The open source CFD toolbox. Available at http://www. openfoam.com. 